\title{
Aerobic training reverses airway inflammation and remodelling in an asthma murine model
}

\author{
R.A. Silva*, R.P. Vieira*, A.C.S. Duarte*, F.D.T.Q.S. Lopes”, A. Perini", T. Mauad", \\ M.A. Martins ${ }^{\#}$ and C.R.F. Carvalho*
}

ABSTRACT: Aerobic training (AT) decreases dyspnoea and exercise-induced bronchospasm, and improves aerobic capacity and quality of life; however, the mechanisms for such benefits remain poorly understood. The aim of the present study was to evaluate the AT effects in a chronic model of allergic lung inflammation in mice after the establishment of airway inflammation and remodelling.

Mice were divided into the control group, AT group, ovalbumin (OVA) group or OVA+AT group and exposed to saline or OVA. AT was started on day 28 for 60 min five times per week for 4 weeks. Respiratory mechanics, specific immunoglobulin $(\mathrm{Ig}) \mathrm{E}$ and $\mathrm{IgG}_{1}$, collagen and elastic fibres deposition, smooth muscle thickness, epithelial mucus, and peribronchial density of eosinophils, CD3+ and CD4+, IL-4, IL-5, IL-13, interferon- $\gamma$, IL-2, IL-1ra, IL-10, nuclear factor (NF)$\kappa B$ and Foxp3 were evaluated.

The OVA group showed an increase in IgE and IgG ${ }_{1}$, eosinophils, CD3+, CD4+, IL-4, IL-5, IL-13, NF- $\kappa B$, collagen and elastic, mucus synthesis, smooth muscle thickness and lung tissue resistance and elastance. The OVA+AT group demonstrated an increase of $\operatorname{IgE}$ and $\operatorname{IgG}_{1}$, and reduction of eosinophils, CD3+, CD4+, IL-4, IL-5, IL-13, NF-KB, airway remodelling, mucus synthesis, smooth muscle thickness and tissue resistance and elastance compared with the OVA group $(p<0.05)$. The OVA+AT group also showed an increase in IL-10 and IL-1ra $(p<0.05)$, independently of Foxp3.

AT reversed airway inflammation and remodelling and T-helper cell 2 response, and improved respiratory mechanics. These results seem to occur due to an increase in the expression of IL-10 and IL-1ra and a decrease of NF-kB.

KEYWORDS: Aerobic training, airway inflammation, airway remodelling, asthma, exercise, rehabilitation

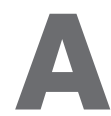
sthma is characterised by chronic airway inflammation and remodelling, hyperresponsiveness and increased levels of T-helper (Th) cell 2 cytokines [1]. Asthmatic airways present with: an increase of eosinophils and subgroups of CD3 Th cells (mainly CD4+ Tcells and mast cells) [1, 2]; mucus hypersecretion; smooth muscle hypertrophy and hyperplasia; increased sub-epithelial deposition of collagen and elastic fibres; and increased epithelial thickness resulting in airway obstruction [3]. The chronic inflammation in asthma is mediated by Th2 cytokines, mainly interleukin (IL)- 4 , IL-5 and IL-13, which play an important role in the maintenance of inflammation and airway remodelling [4]. Th1 cytokines, particularly IL-2 and interferon (IFN)- $\gamma$ are also present in asthmatics [5]; however, its roles in inflammation remains controversial. Some studies demonstrate that Th1 cytokines reduce asthma features [6], although others suggest that Th1 cytokines exacerbate Th2 asthmatic response [5].

Aerobic training (AT) has been used as an important part of the rehabilitation programmes of asthmatic patients [7]. AT decreases dyspnoea, airway hyperresponsiveness, exercise-induced bronchospasm and use of corticosteroids, and improves aerobic capacity and quality of life [7]. The benefits of AT are attributed to an increase in the ventilatory threshold thereby lowering the minute ventilation during mild and moderate exercise [8]. Consequently, breathlessness and the likelihood of provoking exercise induced asthma could be reduced or abolished. AT may also
AFFILIATIONS

Depts of *Physical Therapy (LIM-34)

"Medicine (LIM-20) and

"Pathology (LIM-05), School of

Medicine, University of São Paulo,

São Paulo, Brazil.

CORRESPONDENCE

C.R.F. Carvalho

School of Medicine

University of São Paulo

Av. Dr. Arnaldo 455

1st Floor

Room 1210

01246-903 São Paulo

Brazil

E-mail: cscarval@usp.br

Received:

March 262009

Accepted after revision:

Oct 072009

First published online:

Nov 062009 
decrease the perception of breathlessness through other mechanisms, including respiratory muscle strengthening [9]. However, possible effects of AT mediated through the immune system have been poorly evaluated.

In healthy individuals, AT modulates the immune system by increasing Th1 responses that appear to suppress Th2 [10]. For instance, IFN- $\gamma$ expressed by Th1 lymphocytes of aerobically trained individuals suppresses proliferation of Th2 lymphocytes [11]. This response can be amplified by IL-12 produced by Th1 lymphocytes and antigen presenting cells that enhance IFN- $\gamma$ expression [12]. At present, few studies have investigated the effects of AT on Th2 immune response. PASTVA et al. [13] and VIEIRA and co-workers [14, 15] have shown that AT decreases leukocyte migration to the airway as well as IL-4 and IL-5 expression, immunoglobulin (Ig)E levels and nuclear factor (NF)- $\mathrm{BB}$ expression in asthma animal models. However, a main criticism in those studies is that AT was initiated concomitant to the challenges in airways, which were devoid of significant inflammation and structural changes. This situation may not represent the clinical status of the majority of asthmatics that are submitted to pulmonary rehabilitation.

Therefore, the present study aimed to evaluate the effects of AT performed after the establishment of airway inflammation and remodelling using a chronic model of allergic airway inflammation. We investigated a possible role of NF- $\kappa$ B, Foxp3, Th1 and anti-inflammatory cytokines in mediating these effects. The effects of AT were evaluated on respiratory mechanics, IgE and $\operatorname{IgG}_{1}$ antigen-specific levels, airway eosinophilia and CD3+ and CD4+ lymphocitary inflammation. The expression of Th1 (IL-2 and IFN- $\gamma$ ) and Th2 (IL-4, IL-5 and IL-13) cytokines, as well as regulatory/anti-inflammatory cytokines (IL-10, IL-1ra) and the transcription factors NF- $\mathrm{B}$ and Foxp3, were also evaluated.

\section{MATERIAL AND METHODS \\ Animals}

28 male BALB/c mice (aged 4 weeks and weighing $28 \pm 2$ g) were maintained under standard laboratory conditions with controlled temperature $\left(22 \pm 1^{\circ} \mathrm{C}\right)$ and relative humidity $(40$ $60 \%$ ) on a $12 \mathrm{~h}$ light/dark cycle. They were provided with food and water ad libitum (Labina; Purina, Brazil). The experimental protocol was approved by the ethical committee of the School of Medicine of the University of São Paulo (São Paulo, Brazil). All animal cares and experimental procedures followed the international recommendations of the Helsinki convention for the use and care of animals.

\section{Protocol of chronic allergic lung inflammation}

The mice were assigned to one of four groups $(n=7$ in each group) as follows: control; AT; ovalbumin (OVA); and OVA+AT. The OVA groups received OVA (i.p. $10 \mu \mathrm{g} \cdot$ mouse $^{-1}$ ) diluted in aluminum hydroxide on days $0,14,28$ and 42 as previously described [14-16]. After day 21, mice were exposed to OVA aerosol (1\%); $30 \mathrm{~min}$ per session for three sessions a week until day 54. Control and AT groups were exposed to saline solution.

\section{Physical test and exercise training protocol}

On days 21 to 23 mice were placed on the treadmill (Inbramed, Brazil) for $15 \mathrm{~min}$ at a speed of $0.2 \mathrm{~km} \cdot \mathrm{h}^{-1}$ and a $25^{\circ}$ incline.
On days 25 and 53 the maximal exercise test was performed as previously described [14-16]. Treadmill training (50\% of maximal exercise capacity reached in the physical exercise test) began on day 28 and was performed over 4 weeks, 60 min per session for five sessions a week (fig. 1).

\section{Evaluation of respiratory mechanics}

Mice were anesthetised $\left(50 \mathrm{mg}^{-1} \cdot \mathrm{kg}^{-1}\right.$ i.p. thiopental), tracheostomised and connected to a rodent ventilator (FlexiVent; Scireq, Montreal, QC, Canada) with the tidal volume and frequency set at $10 \mathrm{~mL} \cdot \mathrm{kg}^{-1}$ and $2 \mathrm{~Hz}$, respectively. Oscillatory lung mechanics was performed by producing flow oscillations at different prime frequencies (from 0.25 to $19.625 \mathrm{~Hz}$ ) for $16 \mathrm{~s}$ [17]. Pressure and flow data were obtained and airway impedance was calculated at each frequency [18]. Tissue impedance and elastance (Gtis and $\mathrm{Htis}$, respectively) were obtained by applying the constant-phase model.

\section{IgE and $\operatorname{lgG}_{1}$ anti-OVA antibody titration by passive cutaneous anaphylaxis}

Passive cutaneous anaphylaxis (PCA) was performed in Wistar Furth rats and in BALB/c mice for anti-OVA $\operatorname{IgE}$ and $\operatorname{IgG}_{1}$, respectively. The animals back was shaved and injected intradermally with different serum dilutions. The animals were challenged intravenously with $0.5 \mathrm{mg}$ of OVA in $0.25 \%$ Evans Blue solution, after a sensitisation period of $18-24 \mathrm{~h}$ in rats for $\operatorname{IgE}$ and $2 \mathrm{~h}$ in mice for $\operatorname{IgG}_{1}$ titration. The PCA titre was expressed as the reciprocal of the highest dilution that gave an intradermic allergic reaction $>5 \mathrm{~mm}$ in diameter in duplicate of tests. The detection of threshold of the technique was established at a 1:5 dilution [19].

\section{Lung histology}

$5-\mu \mathrm{m}$ thick sections of the lungs were stained with haematoxylin and eosin to analyse the lung architecture and quantification of airway smooth muscle thickness [14, 20]. Picrosirius was used for bronchial collagen fibre quantification [14, 21], Weigert's resorcin fuchsin with oxidation was used for elastic fibres [22] and Luna was used for detection of peribronchial

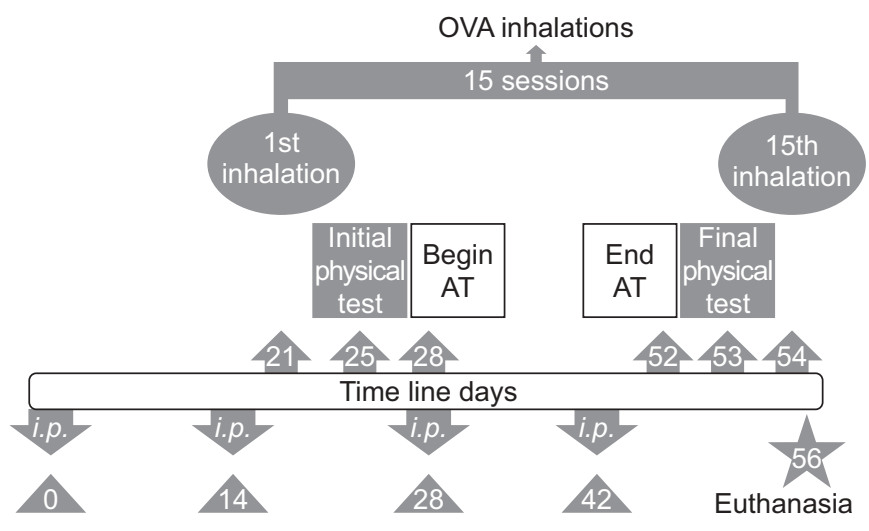

FIGURE 1. Schematic time line of the experimental protocol. Days 0, 14, 28 and 42 represent intraperitoneal (i.p.) injections of ovalbumin (OVA). Days 21 to 54 represent exposure to ovalbumin aerosol. Training adaptation took place from days 21 to 23 , and days 25 and 53 represent the initial and final physical tests. Aerobic training (AT) was initiated on day 28 and ended on day 52. Euthanasia was performed on day 56 . 
eosinophils [14, 23]. Periodic acid-schiff (PAS)-alcian blue (AB) was used to evaluate mucus synthesis $[14,24]$. The slides were coded and the researcher who performed the measurements was unaware of the study groups. Using an Eclipse E-200 microscope (Nikon, Tokyo, Japan), a Cool Snap digital camera (Photometrics, Tokyo) and the image analyses software Image Proplus 4.5 (Media Cybernetics, Bethesda, MD, USA), we evaluated airway smooth muscle thickness, collagen and elastic fibre deposition on the airway wall, and epithelial mucus production. Five airways at $400 \times$ magnification were evaluated for each animal. The area of the smooth muscle layer was quantified by delineating its inner to outer borders. Airway collagen and elastic fibre deposition were evaluated in the area compressed between epithelial basal membranes until airway adventitia. The positive area of collagen and elastic fibres was expressed as a percentage of the total airway wall area. Mucus production was evaluated by counting the epithelial area that was PAS/AB-positive and the results were expressed in percentages [24].

\section{Immunohistochemistry}

$5-\mu \mathrm{m}$ thick sections of the paraffin embedded specimens were used to determinate the peribronchial count of positive inflammatory cells expressing Foxp3, IL-4, IL-5, IL-13, IFN- $\gamma$, IL-2, NF-кB, IL-10, IL-1ra and CD3+ and CD4+ lymphocytes, by immunohistochemistry technique [14]. Briefly, sections were deparaffinised and a $0.5 \%$ peroxidase in methanol solution was applied for $10 \mathrm{~min}$ to inhibit endogenous peroxidase activity. Antigen retrieval was performed with citrate solution for $30 \mathrm{~min}$. Sections were incubated with antiFoxp3 (1:550), anti-IL-4 (1:600), anti-IL-5 (1:700), anti-IL-13 (1:300), anti-IFN- $\gamma(1: 400)$, anti-IL-2 (1:500), anti-NF-кB (1:600), anti-IL-10 (1:700), anti-IL-1ra (1:750), anti-CD3+ (1:500) and anti-CD4+(1:400) (Santa Cruz Biotechnology Inc., Santa Cruz, CA, USA) and left overnight at $48^{\circ} \mathrm{C}$. An ABC Vectastain kit (Vector Elite PK-6105; Vector Laboratories, Burlingame, CA, USA) was used as a secondary antibody and 3,3 diaminobenzidine (Sigma Chemical Co., St Louis, MO, USA) was used as a chromogen. The sections were counterstained with Harris haematoxylin (Merck, Darmstadt, Germany). All primary and secondary antibodies were applied to negative and positive controls. The slides were coded and the researcher who performed the morphometrical analyses was unaware of the study groups. The peribronchial count of positive inflammatory cells for the antibodies described above, and also to CD3+ and CD4+ T lymphocytes and eosinophils, was analysed by using a reticulated ocular of 50 lines and 100 points $(1,000 \times$ magnification; $10,000 \mu \mathrm{m}^{2}$ area) [25]. Cell density was determined as the number of positive marked cells in each field divided by tissue area and expressed as cells $\cdot \mu \mathrm{m}^{-2}$. The results were then transformed to cells $\cdot \mathrm{mm}^{-2}$ by adjusting the units $[25,26]$.

\section{Statistical analysis}

The parametric data were analysed by two-way ANOVA followed by the student Newman-Keuls post hoc test and nonparametric data were analysed by two-way ANOVA followed by the Holm-Sidak post hoc test. Data are presented as mean \pm SD for parametric data and as median (variance) for nonparametric data. The significance level was adjusted to $5 \%$.
Parametric data are presented as bars (figs 2 and 3) and nonparametric data as box plots (figs 4, 5 and 6).

\section{RESULTS}

\section{Exercise capacity}

Control and OVA groups did not present changes in the maximal exercise capacity when we compared the initial and final tests $(\mathrm{p}>0.05)$ (table 1$)$. The AT and OVA+AT groups presented improved exercise capacity when the initial and final tests were compared $(\mathrm{p}<0.01)$ and also when compared with non-trained groups (control and OVA; $\mathrm{p}<0.01$ ).

\section{OVA-specific IgE and IgG,}

OVA sensitisation increased $\operatorname{IgE}$ and $\operatorname{IgG}_{1}$ titres when compared with non-sensitised groups $(\mathrm{p}<0.01)($ table 1$)$ and AT in sensitised animals did not change the $\operatorname{IgE}$ and $\operatorname{IgG}_{1}$ titres when compared with OVA group ( $\mathrm{p}>0.05)$.

\section{Respiratory mechanics}

AT in non-sensitised mice did not change Gtis and Htis when compared with the control group ( $p>0.05$ ) (fig. 2). OVA exposure increased Gtis and Htis when compared with the control and AT groups $(\mathrm{p}<0.05)$. AT performed in sensitised animals (OVA+AT group) resulted in a decrease of Gtis and Htis compared with control levels $(\mathrm{p}<0.05)$ (fig. 2).
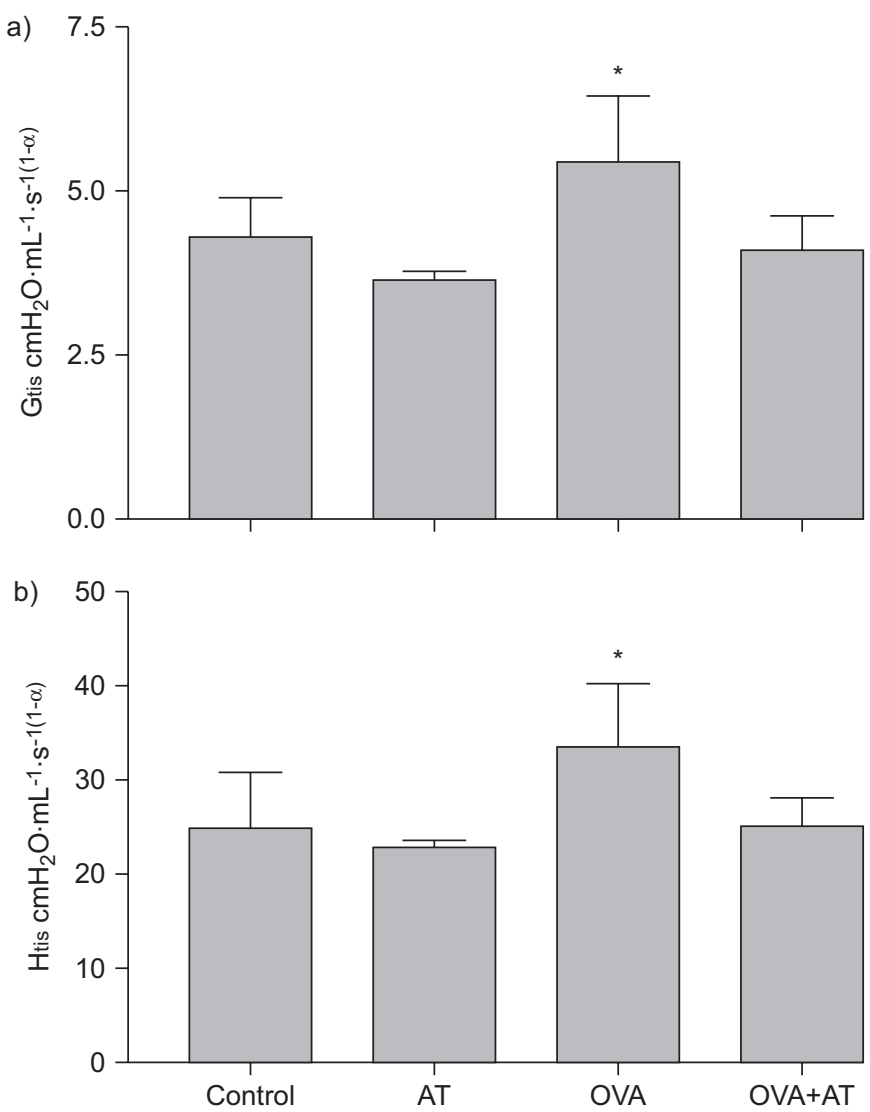

FIGURE 2. Lung mechanics. a) Tissue resistance (Gtis) and b) tissue elastance (Htis). AT: aerobic training; OVA: ovalbumin. Control group: non-sensitised and nontrained; AT group: non-sensitised and AT; OVA group: sensitised and non-trained; OVA+AT: sensitised and AT (control and OVA). Results are presented as mean $\pm \mathrm{SD}$ for all groups. ${ }^{*}: p<0.05$ when compared with other groups. 

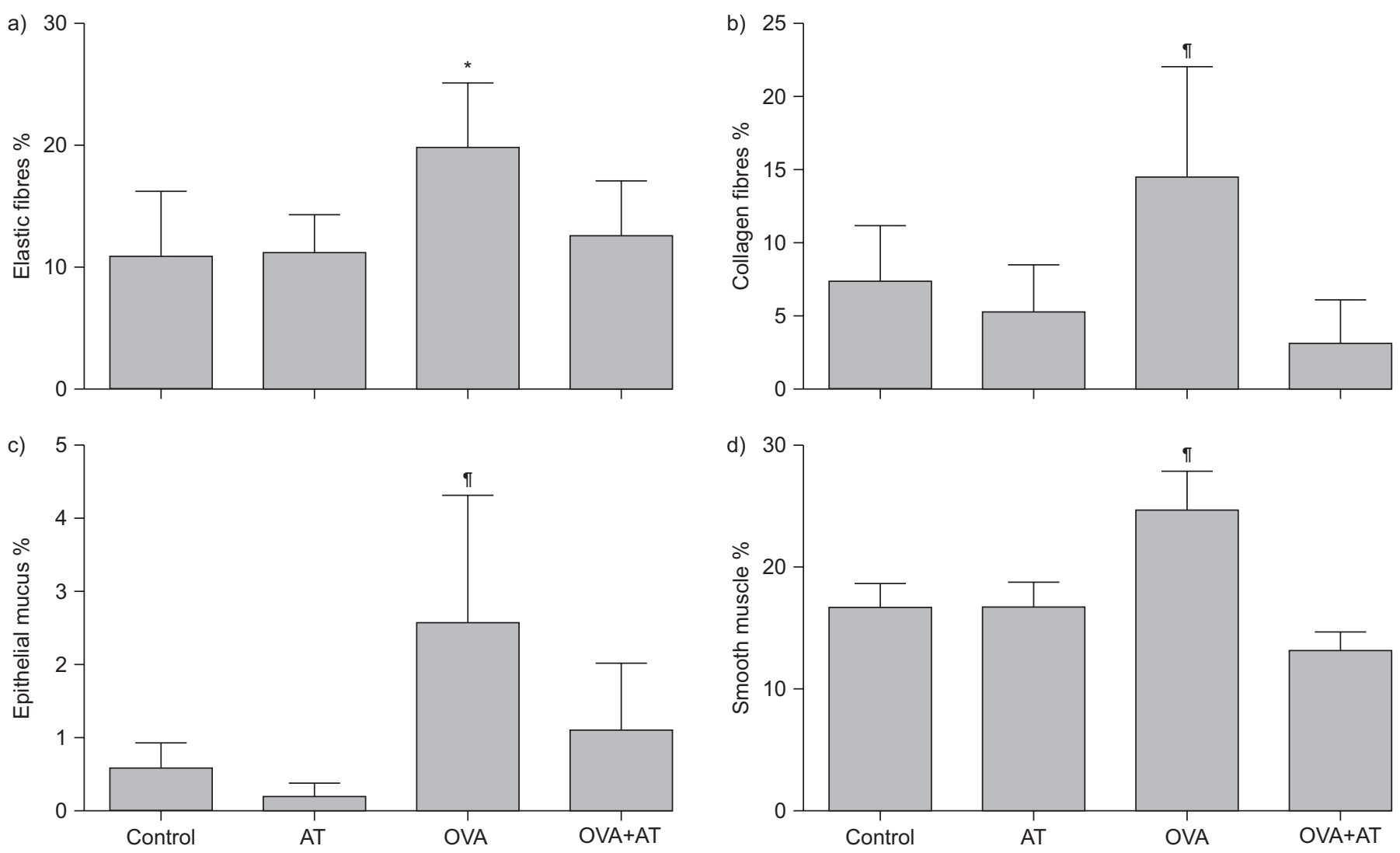

FIGURE 3. a) Elastic fibres, b) collagen fibres, c) epithelial mucus synthesis and d) smooth muscle thickness. AT: aerobic training; OVA: ovalbumin. Control group: nonsensitised and non-trained; AT group: non-sensitised and AT; OVA group: sensitised and non-trained; OVA+AT: sensitised and AT (control and OVA). Results are expressed as mean \pm SD for all groups. *: $p<0.05$ when compared with the control and AT groups; ${ }^{\natural}: p<0.05$ when compared with other groups.

\section{Peribronchial eosinophils, CD3+ and CD4+ T-cells}

AT in non-sensitised animals (AT group) did not change the peribronchial density of eosinophils and CD3+ and CD4+ T lymphocytes compared with the control group ( $p>0.05)$ (fig. 4). Chronic OVA exposure increased the peribronchial density of eosinophils and CD3+ and CD4+ T lymphocytes when compared with the non-sensitised group $(\mathrm{p}<0.05)$. AT in sensitised animals (OVA+AT group) significantly decreased peribronchial eosinophils and CD3+ and CD4+ lymphocytes when compared with the OVA group $(p<0.05)$ and increased when compared with the non-sensitised groups $(\mathrm{p}<0.001)$ (fig. 4).

\section{Peribronchial expression of Th2 cytokines}

AT in non-sensitised animals did not change the peribronchial expression of IL-4, IL-5 and IL-13 when compared with the control group ( $p>0.05$ ) (fig. 5). OVA sensitisation increased the peribronchial expression of IL-4, IL-5 and IL-13 when compared with the non-sensitised group $(\mathrm{p}<0.05)$. The OVA+AT group presented a significant decrease in the peribronchial expression of IL-4, IL-5 and IL-13 ( $\mathrm{p}<0.05)$ when compared with the OVA group (fig. 5). Figure 7 shows the representative photomicrographs of peribronchial expression of IL-5 in all groups.

\section{Peribronchial expression of Th1, regulatory/anti-inflammatory} cytokines IL-10 and IL-1ra, and NF- $\kappa B$ and Foxp3

The expression of IL-2, IFN- $\gamma$, Foxp3, IL-10, IL-1ra and NF- $\kappa B$ was not changed in the AT group compared with the control group ( $p>0.05$ ) (fig. 6). The peribronchial expression of NF- $\mathrm{B}$ was increased in the OVA group when compared with the control group $(\mathrm{p}<0.05)$. However, OVA sensitisation did not change the peribronchial expression of IL-2, IFN- $\gamma$, IL-10 and IL-1ra ( $p>0.05)$. The OVA+AT group presented a decrease in the expression of NF- $\kappa B(p<0.05)$ (fig. $6 c)$, and increased the expression of IL-10 and IL-1ra when compared with the OVA group $(\mathrm{p}<0.05)$. The OVA+AT group did not change the peribronchial expression of IL-2 and IFN- $\gamma$. The expression of Foxp3 was not modified by OVA-induced allergic inflammation or by AT in sensitised animals ( $p>0.05)$.

\section{Airway remodelling}

The AT group did not change airway collagen and elastic fibre deposition, smooth muscle thickness or mucus production when compared with the control group ( $p>0.05$ ) (fig. 3). OVA sensitisation increased airway collagen and elastic fibre deposition, smooth muscle thickness and epithelial mucus production when compared with the control group. The OVA+AT group presented a reduction of airway collagen deposition, smooth muscle thickness and epithelial mucus production when compared with the OVA group $(\mathrm{p}<0.05)$; however, no changes were observed in elastic fibres $(p>0.05)$.

\section{DISCUSSION}

The present study demonstrates that aerobic training reverses OVA-induced peribronchial accumulation of eosinophils, 

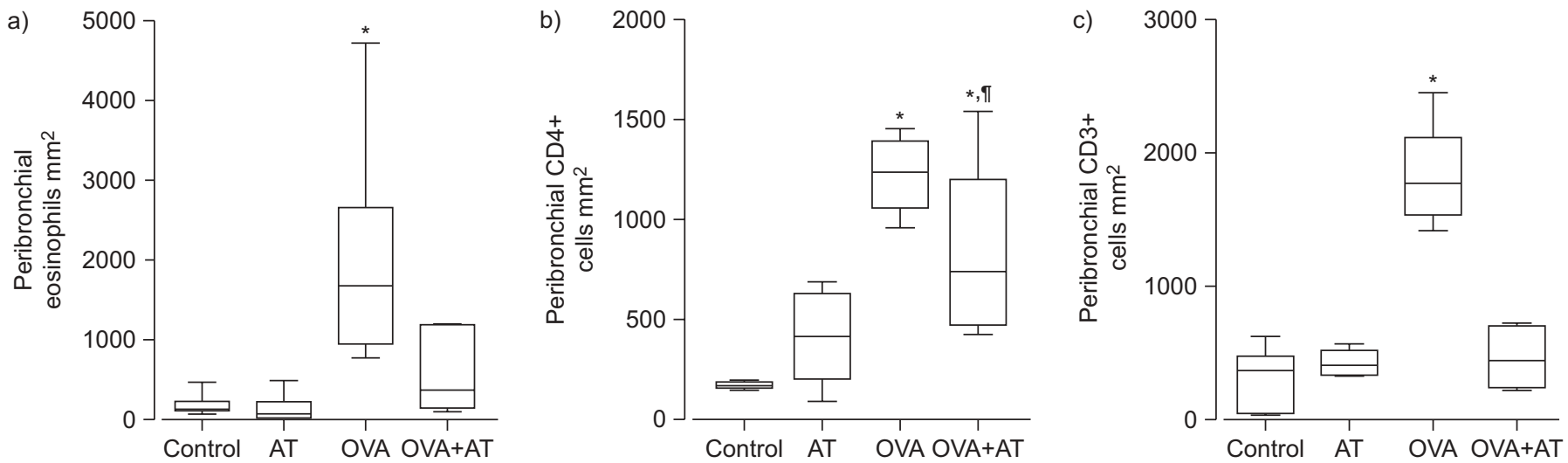

FIGURE 4. Peribronchial density of a) eosinophils, b) CD4+ T lymphocytes and c) CD3+ T lymphocytes. AT: aerobic training; OVA: ovalbumin. Control group: nonsensitised and non-trained; AT group: non-sensitised and AT; OVA group: sensitised and non-trained; OVA+AT: sensitised and AT (control and OVA). Results are presented as mean \pm SD. *: $p<0.05$ when compared with all other groups; ${ }^{\circ}: p<0.05$ when compared with all non-sensitised groups.
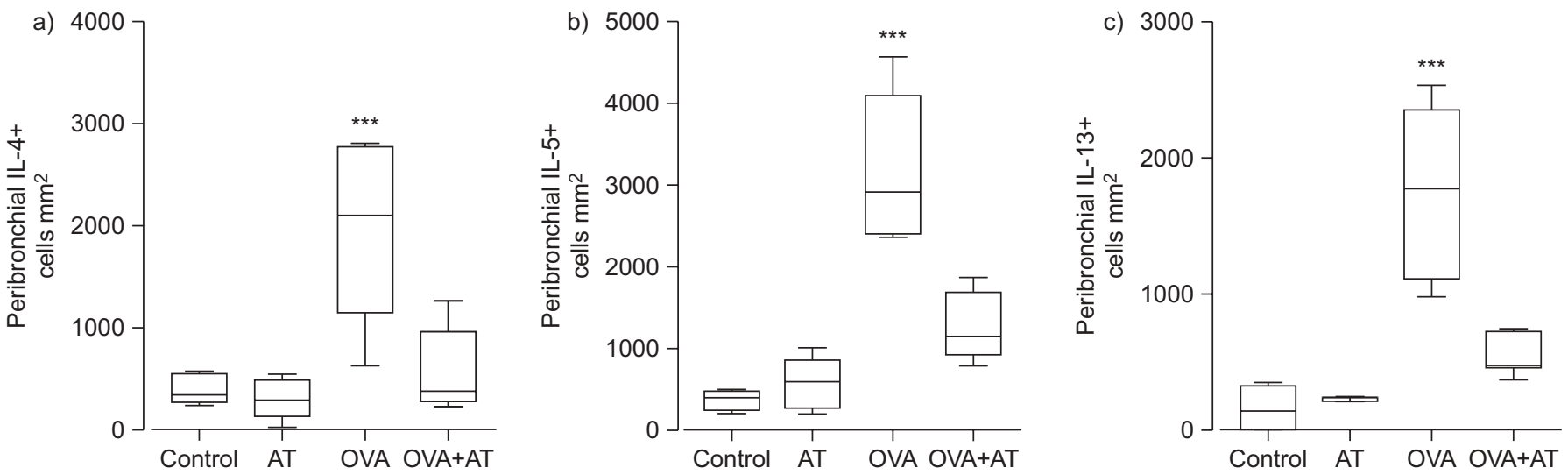

FIGURE 5. Peribronchial expression of T-helper cell 2 cytokines a) interleukin (IL)-4+, b) IL-5+ and c) IL-13+ by inflammatory cells. AT: aerobic training; OVA: ovalbumin Control group: non-sensitised and non-trained; AT group: non-sensitised and AT; OVA group: sensitised and non-trained; OVA+AT: sensitised and AT (control and OVA) Results are presented as mean \pm SD. ${ }^{* * *}: p<0.001$ when compared with other groups.

CD3+ and CD4+ lymphocytes, peribronchial expression of IL-4, IL-5, IL-13, NF- $\kappa$ B, collagen fibre deposition and increase in airway smooth muscle thickness and epithelial mucus production, as well as reduces tissue resistance and elastance. Our results also suggest that these effects are associated with increased expression of anti-inflammatory cytokines IL-10 and IL-1ra.

Asthmatics subjects taking part in an AT programme presented with a reduction in corticosteroids consumption, dyspnoea and exercise-induced bronchospasm, as well as an improvement in the aerobic capacity and health-related quality of life features [7, 9]. Previous experimental studies have investigated the hypothesis that such effects in asthmatic patients could be associated with anti-inflammatory effects of exercise training [13-15]. PASTVA et al. [13] demonstrated that AT decreases neutrophil infiltration in to the airways, IL-4 levels in bronchioalveolar lavage fluid and the expression of NF- $\kappa$; effects that seem mediated, at least partly, by endogenous corticosteroids. VIEIRA and co-workers [14, 15] demonstrated that low or moderate AT intensity also decreases eosinophilic inflammation and IL-4 and IL-5 expression, and these effects are, at least partially, mediated by an increase in the anti-inflammatory cytokine IL-10. Despite the fact that these studies are unique by describing such anti-inflammatory effects in lung allergic inflammation, both models have as a major criticism: the fact that exercise training was initiated simultaneously to the OVA challenges and, thus, prior to the development of airways inflammation and structural changes.

We used a chronic animal model of allergic lung inflammation and the exercise training was started after the establishment of airway inflammation and remodelling. Our results demonstrate that AT reverses airway eosinophilic migration (fig. 4a) and lymphocyte migration (CD3+ and CD4+) (fig. $4 b$ and c). These results are highly relevant as CD3+ and CD4+ T-cells are related to IgE specific synthesis and have a direct effect on the airways through the recruitment of eosinophils [27] and also as a source of pro-inflammatory Th2 cytokines (IL-4, IL-5 and IL-13), which are correlated with the development and maintenance of airway inflammation and remodelling [4].

For instance, IL-4 seems to perpetuate mast cell activation, which is responsible for $\operatorname{IgE}$ and $\operatorname{IgG}_{1}$ release, eosinophils 

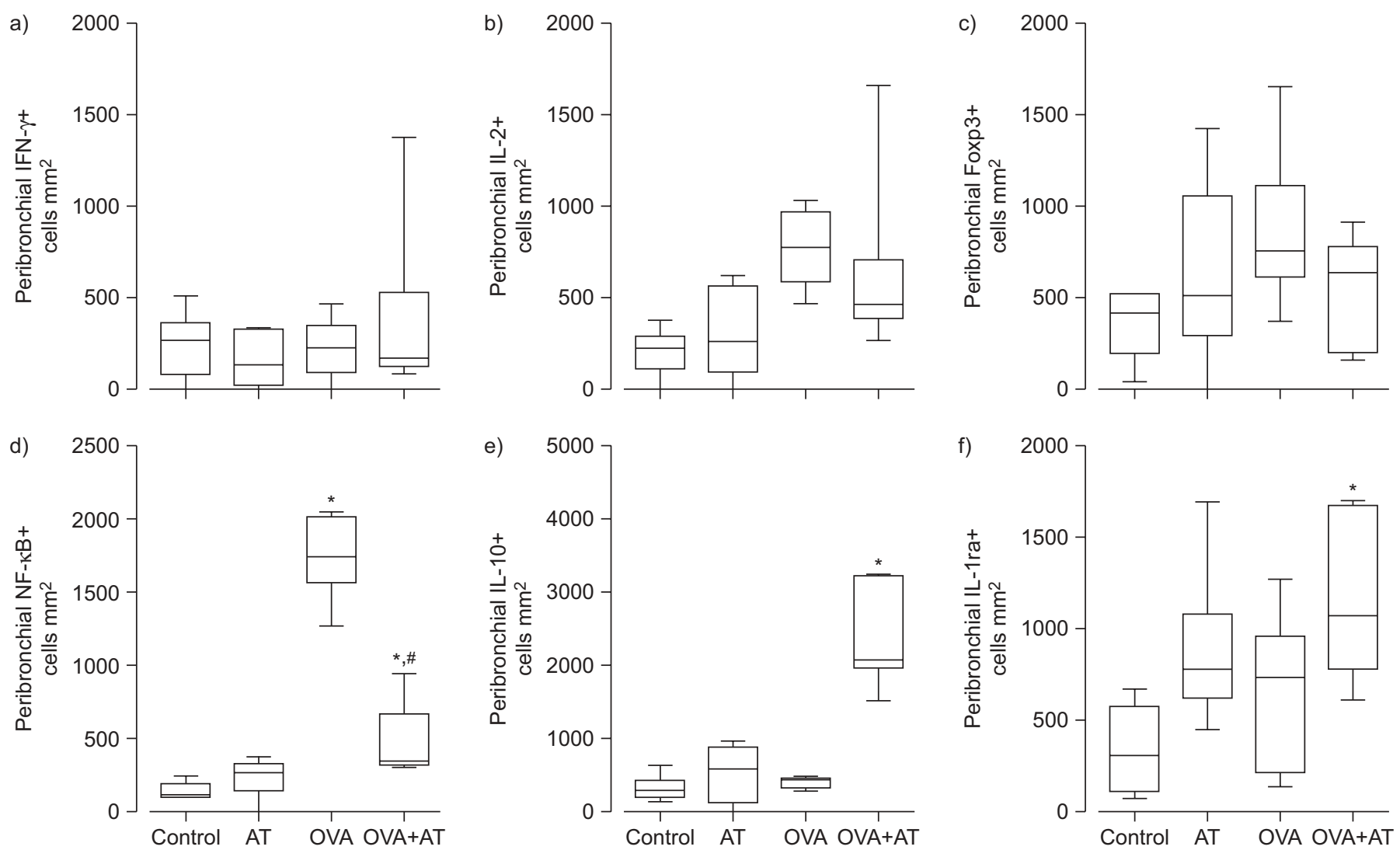

FIGURE 6. Peribronchial density of a) interferon (IFN)- $\gamma+$, b) interleukin (IL)-2+, c) Foxp3+, d) nuclear factor (NF)-kB, e) IL-10+ and f) IL-1ra+. AT: aerobic training; OVA: ovalbumin. Control group: non-sensitised and non-trained; AT group: non-sensitised and AT; OVA group: sensitised and non-trained; OVA+AT: sensitised and AT (control and OVA). Results are expressed as mean \pm SD. ${ }^{*}: p<0.05$ when compared with other groups; ${ }^{*}: p<0.05$ when compared with the control and AT groups.

activation and recruitment, mucus production and play a role in Th2 maintenance $[1,2,28]$. IL-5 stimulates the proliferation, differentiation, migration, survival and activation of eosinophils in the bone marrow and is a chemoattractant to eosinophils $[4,26]$ reducing the inhibitory effects of IL-2 on eosinophils migration [29]. IL-13 causes increased levels of mucus production by airway epithelial cells [4, 24]. Our study showed that AT decreased the peribronchial expression of IL-4, IL- 5 and IL-13 by inflammatory cells, predominantly by mononuclear cells (fig. 5). These data reinforce that AT is capable of reversing the Th2 immune response in a murine model of asthma.

Airway remodelling is defined as the presence of structural changes in the airways with increased deposition of collagen and elastic fibres and other extracellular matrix proteins, smooth muscle hyperplasia and hypertrophy, and goblet cells hyperplasia followed by excessive mucus secretion [3].

\section{TABLE 1 Weight, physical test performance and titres of immunoglobulin (Ig)}

\begin{tabular}{|c|c|c|c|c|c|c|}
\hline \multirow[t]{2}{*}{ Groups } & \multicolumn{2}{|c|}{ Weight $\mathbf{g}$} & \multicolumn{2}{|c|}{ Physical test min } & \multicolumn{2}{|c|}{ Specific OVA antibodies Log } \\
\hline & Initial & Final & Initial & Final & $\lg E$ titre & $\lg G_{1}$ titre \\
\hline AT & $28.5 \pm 1.5$ & $29.1 \pm 1.3$ & $35.8 \pm 4.9$ & $44.8 \pm 4.4^{\star \star}$ & $0.0 \pm 0$ & $0.0 \pm 0$ \\
\hline OVA & $26.8 \pm 1.6$ & $27.9 \pm 0.6$ & $35.3 \pm 2.8$ & $29.8 \pm 2.3$ & $5.1 \pm 0.4^{\star \star \star}$ & $7.3 \pm 0^{\star * *}$ \\
\hline OVA + AT & $28.1 \pm 1.7$ & $28.3 \pm 1.8$ & $31.8 \pm 3.5$ & $39.8 \pm 3.4^{\star \star}$ & $4.8 \pm 0.5^{\star \star \star}$ & $7.8 \pm 0.7^{\star \star \star}$ \\
\hline
\end{tabular}

Data are presented as mean \pm SD. AT: aerobic training; OVA: ovalbumin. Control group: non-sensitised and non-trained; AT group: non-sensitised and AT; OVA group: sensitised and non-trained; OVA+AT: sensitised and AT (control and OVA). **: $p<0.01$ compared with non-trained groups (control and AT); ***: $p<0.001$ compared with non-sensitised groups (control and AT). 


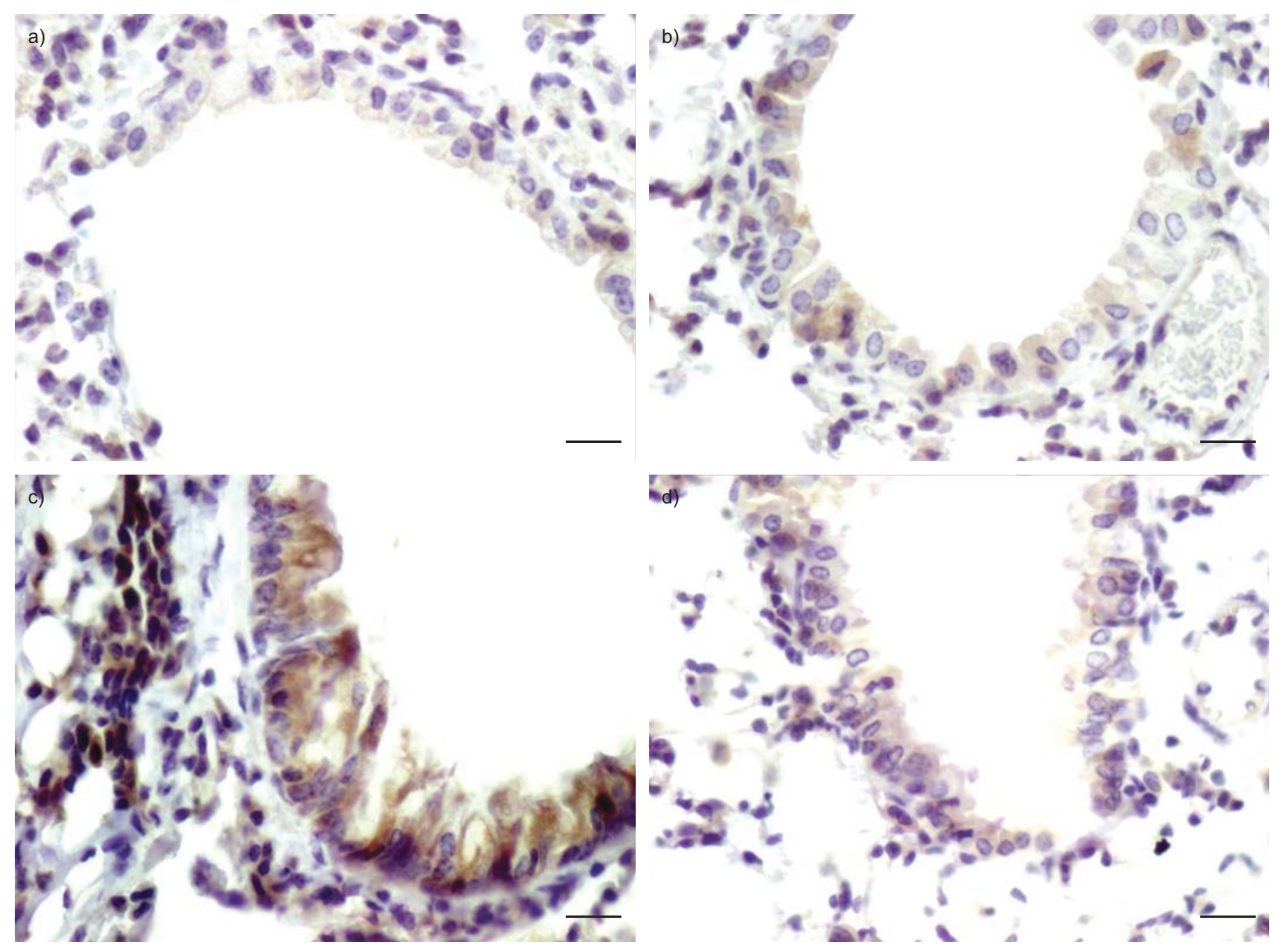

FIGURE 7. Photomicrographs of peribronchial expression of interleukin (IL)- 5 by inflammatory cells. Note that the majority of cells expressing IL-5 are mononuclear cells a) Ovalbumin (OVA) group (sensitised and non-trained), b) OVAt aerobic training (AT) group (sensitised and AT (control and OVA)), c) control group (non-sensitised and nontrained), and d) AT group (non-sensitised and AT). Scale bars $=25 \mu \mathrm{m}$.

Previous studies using animal models have shown that after 30 days exposure to OVA inhalation mice present with airway remodelling [30-32]. In the present study, the AT was started after 28 days of OVA inhalation and our results demonstrate that AT reduces OVA-induced airway remodelling, including collagen fibre deposition, smooth muscle thickness and mucus production in this murine model of asthma (fig. 3). These data are relevant since no previous studies have demonstrated that AT can reverse airway inflammation and remodelling. Our murine model of asthma also resulted in an increased tissue resistance and elastance, which was completely reversed by AT (fig. 2). Our results reinforce previous findings by showing that changes in lung mechanics in sensitised mice seem to be related to airway inflammation and remodelling [31-33].

We evaluated three possible mechanisms through which exercise could modulate the observed effects: 1) by increasing Th1 response (IL-2 and IFN- $\gamma$ ) [34]; 2) by changing the expression of modulatory cytokines (IL-10 and IL-1ra) [35]; and 3) through modulating NF-кB and Foxp3 [36-38]. We observed that AT did not change the expression of IL-2 and
IFN- $\gamma$ either in non-sensitised or in sensitised mice (fig. 7a and b), suggesting that the beneficial effects of AT in our murine model of asthma are not mediated by an improvement of Th1 response. We also observed that $\mathrm{AT}$ in sensitised animals reduced the peribronchial expression of NF- $\kappa$ B by inflammatory cells suggesting that it could be involved in both antiinflammatory and anti-fibrotic effects induced by AT. In addition, we also showed that AT increased the peribronchial expression of expression of anti-inflammatory cytokines IL-10 and IL-1ra. We showed that Foxp3 levels were unchanged either after OVA inflammation or by exercise, suggesting that the increase in IL-10 in our animal model seems to occur independently of Foxp3 regulation.

The clinical benefits induced by AT in asthmatics subjects [7, 39] have been explained by the reduction in ventilatory threshold [8]. Our study is the first to evaluate the effect of AT after several OVA inhalations to simulate airway inflammation, as occurs in asthmatics patients. Our results might suggest that improvement in AT can modulate airway allergic inflammation. Interestingly, a recent study showed that 
asthmatic females with higher physical fitness present with less episodes of asthma exacerbations. The authors hypothesised that an increase in physical fitness should be used as adjuvant treatment in asthma [40], which is supported by our findings.

\section{Conclusion}

We conclude that AT reverses airway inflammation and remodelling, improves respiratory mechanics and reduces Th2 immune response in a murine model of asthma. These effects seem to occur in response to a decreased expression of $\mathrm{NF}-\kappa \mathrm{B}$ and an increased expression of anti-inflammatory cytokines IL-10 and IL-1ra. Our data fully support the "emerging concept" that physical fitness should be used as adjuvant treatment in asthma.

\section{SUPPORT STATEMENT}

T. Mauad, C.RF. Carvalho and M.A. Martins are Senior Researchers from Conselho Nacional de Desenvolvimento Científico e Tecnológico. This study was funded by CAPES, Fundação de Amparo à Pesquisa do Estado de São Paulo and Laboratório Investigação Médica (LIM-20), Clinics Hospital, School of Medicine (University of São Paulo, São Paulo, Brazil).

\section{STATEMENT OF INTEREST}

None declared.

\section{REFERENCES}

1 Holgate S. Pathogenesis of asthma. Clin Exp Allergy 2008; 38: 872897.

2 Galli SJ, Tsai M, Piliponsky AM. The development of allergic inflammation. Nature 2008; 24: 445-454.

3 Fixman ED, Stewart A, Martin JG. Basic mechanisms of development of airway structural changes in asthma. Eur Respir J 2007; 29: 379-389.

4 Nath P, Leung SY, Williams AS, et al. Complete inhibition of allergic airway inflammation and remodelling in quadruple IL-4/ 5/9/13?/? mice. Clin Exp Allergy 2007; 37: 1427-1435.

5 Corrigan CJ, Kay AB. CD4 T lymphocyte activation in acute severe asthma. Int Arch Allergy Appl Immunol 1991; 94: 270-271.

6 Tang C, Inman MD, van Rooijen N, et al. Th type 1-stimulating activity of lung macrophages inhibits Th2-mediated allergic airway inflammation by an IFN- $\gamma$-dependent mechanism. $J$ Immunol 2001; 166: 1471-1481.

7 Fanelli A, Cabral AL, Neder JA, et al. Exercise training on disease control and quality of life in asthmatic children. Med Sci Sports Exerc 2007; 39: 1474-1480.

8 Clark C, Cochrane L. Assessment of work performance in asthma for determination of cardiorespiratory fitness and training capacity. Thorax 1988; 43: 745-749.

9 Ram FSF, Robinson SM, Black PN. Effects of physical training in asthma: a systematic review. Br J Sports Med 2000; 34: 162-167.

10 Lakier Smith L. Overtraining, excessive exercise, and altered immunity: is this a $\mathrm{T}$ helper-1 versus $\mathrm{T}$ helper-2 lymphocyte response? Sports Med 2003; 33: 347-364.

11 Fernandez-Botran R, Sanders VM, Mosmann TR, et al. Lymphokine mediated regulation of the proliferative response of clones of T helper 1 and T helper 2 cells. J Exp Med 1988; 168 543-558

12 Heufler C, Koch F, Stanzl U, et al. Interleukin-12 is produced by dendritic cells and mediates T helper 1 cell development as well as interferon-gamma production by $\mathrm{T}$ helper 1 cells. Eur J Immunol 1996; 26: 659-668.
13 Pastva A, Estell K, Shoeb TR, et al. Aerobic exercise attenuates airway inflammation in a mouse model of atopic asthma. J Immunol 2004; 172: 4520-4526.

14 Vieira RP, Claudino RC, Duarte AC, et al. Aerobic exercise decreases chronic allergic lung inflammation and airway remodeling in mice. Am J Respir Crit Care Med 2007; 176: 1-7.

15 Vieira RP, de Andrade VF, Duarte AC, et al. Aerobic conditioning and allergic pulmonary inflammation in mice II: effects on lung vascular and parenchymal inflammation and remodeling. Am J Physiol Lung Cell Mol Physiol 2008; 295: L670-L679.

16 Vieira RP, Claudino RC, Duarte AC, et al. Creatine supplementation exacerbates allergic lung inflammation and remodeling in mice. Am J Respir Cell Mol Biol 2007; 37: 660-667.

17 Fust A, LeBellego F, Iozzo RV, et al. Alterations in lung mechanics in decorin-deficient mice. Am J Physiol Lung Cell Mol Physiol 2005; 288: L159-L166.

18 Hantos Z, Collins RA, Turner DJ, et al. Tracking of airway and tissue mechanics during TLC maneuvers in mice. J Appl Physiol 2003; 95: 1695-1705.

19 Mota I, Perini A. A heat labile mercaptoethanol susceptible homocytotropic antibody in the guinea pig. Life Science 1970; 9: 923-930.

20 Arantes-Costa FM, Lopes FDTQS, Toledo AC, et al. Effects of residual oil fly ash (ROFA) in mice with chronic allergic pulmonary inflammation. Toxicol Pathol 2008; 36: 680-686.

21 Montes GS. Structural biology of the fibres of the collagenous and elastic systems. Cell Biol Int 1996; 20: 15-27.

22 Dolhnikoff M, Mauad T, Ludwig MS. Extracellular matrix and oscillatory mechanics of rat lung parenchyma in bleomycininduced fibrosis. Am J Respir Crit Care Med 1999; 160: 1750-1757.

23 Ying S, Meng Q, Smith SJ, et al. Methods for identifying human eosinophils in blood and tissues. Allergy Clin Immunol Int 2002; 14: 64-71.

24 Cho JY, Miller M, Baek KJ, et al. Inhibition of airway remodeling in IL-5-deficient mice. J Clin Invest 2004; 113: 551-560.

25 Lancas T, Kasahara D, Prado CM, et al. Comparison of early and late responses to antigen of sensitized guinea pig parenchymal lung strips. J Appl Physiol 2006; 100: 1610-1616.

26 Nakajima H, Iwamoto I, Tomoe S, et al. CD4+ T-lymphocytes and interleukin-5 mediate antigen-induced eosinophil infiltration into the mouse trachea. Am Rev Respir Dis 1992; 146: 374-377.

27 Lampinen M, Carlson M, Venge HP. Cytokine-regulated accumulation of eosinophils in inflammatory disease. Allergy 2004; 59: 793-805.

28 Carlson M, Peterson C, Venge P. The influence of IL-3, IL-5 and GMCSF on normal human eosinophil and neutrophil C3b-induced degranulation. Allergy 1993; 48: 437-442.

29 Levi-Schaffer F, Barkans J, Newman TM, et al. Identification of interleukin-2 in human peripheral blood eosinophils. Immunology 1996; 87: 155-161.

30 Hewitt M, Creel A, Estell K, et al. Acute exercise decreases airway inflammation, but not responsiveness, in an allergic asthma model. Am J Respir Cell Mol Biol 2009; 40: 83-89.

$31 \mathrm{Kim} \mathrm{SJ}, \mathrm{Kim} \mathrm{CH}$, Ahn $\mathrm{JH}$, et al. Time sequence of airway remodeling in a mouse model of chronic asthma: the relation with airway hyperresponsiveness. J Korean Med Sci 2007; 22: 183-191.

32 Locke NR, Royce SG, Wainewright JS, et al. Comparison of airway remodeling in acute, subacute, and chronic models of allergic airways disease. Am J Respir Cell Mol Biol 2007; 36: 625-632.

33 Nakashima AS, Prado CM, Lanças T, et al. Oral tolerance attenuates changes in vitro lung tissue mechanics and extracellular matrix remodeling induced by chronic allergic inflammation in guinea pigs. J Appl Physiol 2008; 104: 1778-1785.

34 Ray A, Cohn L. Altering the Th1/Th2 balance as a therapeutic strategy in asthmatic diseases. Curr Opin Investig Drugs 2000; 1 : 442-448. 
35 Nakagome K, Dohi M, Okunishi K, et al. In vivo IL-10 gene delivery suppresses airway eosinophilia and hyperreactivity by down-regulating APC functions and migration without impairing the antigen-specific systemic immune response in a mouse model of allergic airway inflammation. J Immunol 2005; 174: 6955-6966.

36 Das J, Chen $\mathrm{CH}$, Yang L, et al. A critical role for NF- $\mathrm{kB}$ in Gata3 expression and Th2 differentiation in allergic airway inflammation. Nat Immunol 2001; 2: 45-50.

37 Bettelli E, Dastrange M, Oukka M. Foxp3 interacts with nuclear factor of activated T cells and NF-kappa B to repress cytokine gene expression and effector functions of T helper cells. Proc Natl Acad Sci USA 2005; 102: 5138-5143.

$38 \mathrm{Wu} \mathrm{K}, \mathrm{Bi} \mathrm{Y}$, Sun $\mathrm{K}$, et al. IL-10-producing type 1 regulatory $\mathrm{T}$ cells and allergy. Cell Mol Immunol 2007; 4: 269-275.

39 Neder JA, Nery LE, Silva AC, et al. Short term effects of aerobic training in the clinical management of moderate to severe asthma in children. Thorax 1999; 54: 202-206.

40 Garcia-Aymerich J, Varraso R, Antó JM, et al. Prospective study of physical activity and risk of asthma exacerbations in older women. Am J Respir Crit Care Med 2009; 179: 999-1003. 